\title{
Stern-Gerlach-like approach to electron orbital angular momentum measurement
}

\author{
Tyler R. Harvey, ${ }^{1, *}$ Vincenzo Grillo, ${ }^{1,2,3}$ and Benjamin J. McMorran ${ }^{1}$ \\ ${ }^{1}$ Department of Physics, University of Oregon, Eugene, Oregon 97403, USA \\ ${ }^{2}$ CNR-Istituto Nanoscienze, Centro S3, Via G Campi 213/a, I-41125 Modena, Italy \\ ${ }^{3}$ CNR-IMEM Parco Area delle Scienze 37/A, I-43124 Parma, Italy
}

(Received 17 June 2016; published 28 February 2017)

\begin{abstract}
Many methods now exist to prepare free electrons into orbital-angular-momentum states, and the predicted applications of these electron states as probes of materials and scattering processes are numerous. The development of electron orbital-angular-momentum measurement techniques has lagged behind. We show that coupling between electron orbital angular momentum and a spatially varying magnetic field produces an angular-momentum-dependent focusing effect. We propose a design for an orbital-angular-momentum measurement device built on this principle. As the method of measurement is noninterferometric, the device works equally well for mixed, superposed, and pure final orbital-angular-momentum states. The energy and orbital-angular-momentum distributions of inelastically scattered electrons may be simultaneously measurable with this technique.
\end{abstract}

DOI: 10.1103/PhysRevA.95.021801

(a) Introduction. How does one measure the orbital angular momentum (OAM) of the quantum state of an unbound, massive, charged particle after interaction with another particle or a material? Free electrons with OAM, also called electron vortices, are now routinely prepared in electron microscopes [1-11] and control of this new degree of freedom is widely recognized as a useful tool in the both the study of materials and basic physical processes [2,12-14]. A variety of impressive techniques now exist to prepare an electron in an OAM state. Full control of free electron orbital angular momentum, though, demands good measurement tools.

One of the most promising potential applications of electron OAM-measurement of magnetization at atomic resolution via helical dichroism spectroscopy-serves as an excellent example of the importance of both preparation and postselection in applications of electron OAM. Magnetic dichroism has, surprisingly, not yet been realized with electrons prepared in OAM states. This application is analogous to $\mathrm{x}$-ray magnetic circular dichroism (XMCD), a widely used technique for magnetization measurement based on the ratios of coretransition peaks in left- and right-circularly polarized x-ray absorption spectra. There exists a crucial difference, though, between circular dichroism, which involves controlled transfer of photon spin angular momentum, and helical dichroism, which involves controlled transfer of electron OAM [14]. Photons are massless and can be absorbed by materials, so the final state of a photon in a circular dichroism measurement is just the vacuum state. Electrons are massive, and carry away nonzero energy and angular momentum from an interaction. If we seek to gain the most information about a material in an electron spectroscopy experiment, we ought to measure both

\footnotetext{
*Author to whom correspondence should be addressed: trh@uoregon.edu

Published by the American Physical Society under the terms of the Creative Commons Attribution 4.0 International license. Further distribution of this work must maintain attribution to the author(s) and the published article's title, journal citation, and DOI.
}

the final electron energy and OAM [15]. Helical dichroism can be made far more efficient with careful postselection of electron OAM states. This insight, in fact, applies to many applications of electron OAM.

There are a wide range of applications of good OAM postselection. Theoretical predictions and simulation suggest that electron impact ionization [16,17], photoionization [18,19], electron-atom scattering [20], material investigation with angle-resolved photoelectron spectroscopy [21] and electron energy loss spectroscopy [22], production of spin-polarized electrons [23], and even high-energy elementary particle collisions [24] can produce nontrivial final OAM states and could therefore benefit from OAM postselection.

Several techniques have so far been developed for electron OAM measurement; they work well as quality-assurance tests for new OAM state preparation techniques. All have limitations that prohibit their application to postselection of a single final state of an inelastic interaction. Indeed, two recent theoretical proposals that demand perfect postselection of OAM final states for application of electron OAM to the study of materials recognized that existing techniques are not sufficient [13,25]. Self-interferometric techniques [5,26-28] depend on analysis of the spatial distribution of an electron after a transformation. In general, inelastic interaction of an electron and a material produces mixed electron final states thanks to entanglement with the material. Mixed and superposed OAM states are extremely difficult to quantitatively measure with self-interferometric techniques [29]. Furthermore, energy-filtered TEM is necessary to isolate and analyze the spatial distribution of the states scattered to a given energy. Holographic phase-flattening [30,31] can partially spatially isolate a single component of a mix of inelastically scattered final OAM states, but is currently fairly inefficient.

We propose a technique for OAM postselection based on coupling of OAM to a spatially varying magnetic field. The effect is analogous to the coupling between spin and a spatially varying magnetic field that Stern and Gerlach employed in their demonstration of the quantization of spin [32]. In the Stern-Gerlach device, spins aligned (antialigned) with the magnetic field are pulled by the Zeeman interaction toward 
the side of the device with higher (lower) field strength. Unlike the Stern-Gerlach device for measurement of spin, we consider a cylindrically symmetric design for measurement of OAM. Cylindrical symmetry guarantees conserve electron OAM through the measurement device [33] and control the Lorentz force [34,35]. Fortunately, cylindrically symmetric, spatially varying magnetic fields find great use as electron round lenses [36]. We show that the coupling of OAM to the field of a magnetic round lens produces a shift in the focal length of a magnetic round lens. In this proposed device, electrons with orbital dipole moments aligned (antialigned) with the magnetic field are pulled inward toward (pushed outward away from) the strong magnetic field along the optic axis.

(b) OAM-dependent focusing effect. An ideal measurement device introduces a unitary interaction that entangles a state of interest with a measurement apparatus state that lies in a separate Hilbert space [37]. In the case of electron orbitalangular-momentum measurement, we consider entangling orbital-angular-momentum states (the states of interest) with radial states (the measurement apparatus state). In other words, we seek to encode information about orbital angular momentum in the radial wave function of the electron and then measure the position of the electron. The simplest realization of such a measurement device is an orbital-angular-momentumdependent lensing effect.

For a state propagating along the $z$ axis, the transfer function of a lens with focal length $f$ on an electron with wavelength $\lambda$ is

$$
U_{\text {lens }}=e^{-i \pi \rho^{2} / \lambda f},
$$

where $\rho$ is the distance from the $z$ axis in cylindrical coordinates $(z, \rho, \phi)$.

If, instead, we want an OAM-dependent focal length, we will want to construct a transfer function

$$
U=\exp \left(-i \frac{L_{z} \rho^{2}}{\hbar b^{2}}\right)
$$

This transfer function produces a quantum nondemolition measurement of orbital angular momentum: OAM is an eigenstate of both this transfer function and the free-space Hamiltonian, and OAM is encoded in the radial distribution of the electron wave function. The effect of this transfer function on an OAM state is visualized in Fig. 1.

For quick insights into the potentials necessary to produce an OAM-dependent lensing effect, we notice that, in the short-time limit, the time evolution operator applies a phase modulation that is proportional to the Hamiltonian. (See Sec. I of the Supplemental Material [38] for an explanation of a magnetic round lens in these terms.) So, we need to find a potential that will produce an $L_{z} \rho^{2}$ term in the electron Hamiltonian.

Counting powers of momentum and position, we see that only the $\mathbf{A} \cdot \mathbf{p}$ term can produce this term. In particular, we can see that we will produce a transfer function like (2) with the vector potential

$$
\mathbf{A}=\left(B_{1}(z) \frac{\rho}{2}-B_{3}(z) \frac{\rho^{3}}{8 b^{2}}+\ldots O\left(\rho^{5}\right)\right) \hat{\phi},
$$

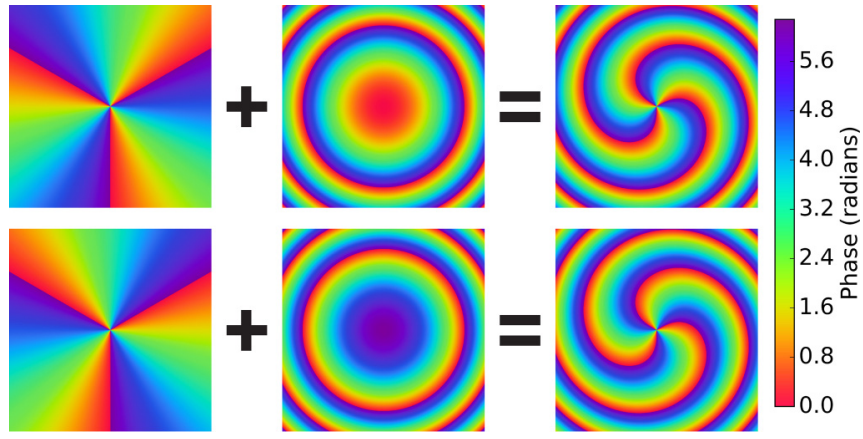

FIG. 1. Illustration of the effect of the orbital-angularmomentum-dependent lensing effect. Propagation of a wave with, in this case, $\pm 3 \hbar$ OAM, and therefore an azimuthal phase (first column), in a Hamiltonian with the OAM-dependent lensing term in Eq. (4) produces a parabolic phase (second column) in proportion to the OAM. The result is a spiraling phase with a winding magnitude and direction that depends on OAM (third column).

where $B_{1}$ and $B_{3}$ describe the longitudinal profile of the field, i.e., $B_{z}=B_{1}(z)-B_{3}(z) \frac{\rho^{2}}{2 b^{2}}$. This vector potential corresponds to a magnetic field that points along the $\pm \hat{\mathbf{z}}$ direction at the origin and curves outward away from the origin over a length scale $b$. We will call $b$ the dispersion length. The corresponding Hamiltonian for an electron in this vector potential includes two lensing terms,

$$
H_{\mathrm{lens}}=\frac{1}{8 m_{e}}\left(e^{2} B_{1}^{2}-\frac{e B_{3} L_{z}}{b^{2}}\right) \rho^{2},
$$

where $e=|e|$ is the magnitude of the electron charge. The latter term produces an OAM dependence in the focal length of the lensing effect. Figure 2 illustrates this lensing effect with multislice-simulated [39] propagation of superposed OAM states in this Hamiltonian [40] and ray trajectories calculated by numerical integration of the radial equation of motion. Filled intervals represent a range of classical trajectories that correspond to a single wave function.

The intuitive explanation in terms of the Zeeman potential $U_{Z}=-\boldsymbol{\mu} \cdot \mathbf{B}$ shows us that a magnetic dipole $\boldsymbol{\mu}$ aligned with a magnetic field $\mathbf{B}$ is pulled toward regions of higher field strength by a force $F_{\rho}=-\frac{\partial U_{Z}}{\partial \rho}$. An orbital magnetic dipole $\boldsymbol{\mu}=-\frac{e}{2 m_{e}} \mathbf{L}$ is therefore pulled inward in a field when the longitudinal strength $B_{z}(z, \rho)$ decreases away from the optic axis (i.e., $B_{3}$ is nonzero) and the orbital dipole moment is aligned with the field. Although we treat only spin-unpolarized electrons here, it may be interesting in the future to examine the analogous effect on spin-polarized electrons. We expect that the OAM-dependent lensing effect, a spin-dependent lensing effect, and spin-to-orbital conversion via spin precession $[23,41]$ should all be independently observable in the nonrelativistic limit.

The vector potential (3) above is an approximation to the vector potential of any cylindrically symmetric current distribution with azimuthal current flow. The dispersion length is related to the radial extent of the current distribution. In the case of a single loop of wire of radius $R, b$ is just $R$ and $B_{3}$ is on the same order as $B_{0}$, the magnetic field at the center of the loop. So, in fact, there exists a small OAM dependence in the 


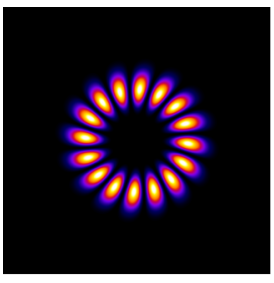

(a)

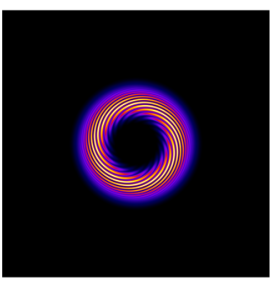

(d)



(g)

FIG. 2. Top: Multislice-simulated intensities of a superposition of $m= \pm 8$ Laguerre-Gaussian orbital-angular-momentum states with $E=80 \mathrm{keV}$ in a Glaser-model, i.e., Eq. (50) in the Supplemental Material [38], field with maximum field strength $B_{0}=2 \mathrm{~T}$, longitudinal extent $a=1 \mathrm{~mm}$, and an OAM dispersion length $b=79 \mathrm{~nm}$, sampled at (a) $-2.0 \mathrm{~mm}$, (b) $-1.5 \mathrm{~mm}$, (c) $-1.0 \mathrm{~mm}$, (d) $-0.5 \mathrm{~mm}$, (e) $0.0 \mathrm{~mm}$, and (f) $0.5 \mathrm{~mm}$ from the center of the lens. Bottom: Ray trajectories for $m=-8, m=0$, and $m=+8$ modes calculated by numerical integration of the radial equation of motion corresponding to the full Hamiltonian in Eq. (16) in the Supplemental Material [38] with a Glaser-model field.

focal length of any standard magnetic round lens. The key to designing an orbital-angular-momentum measurement device is to isolate or maximize the OAM dependence.

To do anything with an OAM-dependent lensing effect, we need to know the focal length of the lens. The focal length can be calculated analytically with two reasonable approximations: the paraxial approximation and the thin lens approximation. The thin lens-paraxial Schrödinger equation [42] for an electron in our model vector potential (3) is a separable first-order differential equation,

$$
2 i k_{z} \frac{\partial \chi}{\partial z}=\frac{e}{\hbar}\left(B_{1}(z)-B_{3}(z) \frac{\rho^{2}}{4 b^{2}}\right) m \chi+\frac{e^{2} B_{1}^{2}(z)}{4 \hbar^{2}} \rho^{2} \chi
$$

for a transverse wave function $\chi(\rho, \phi)$ with $L_{z} \chi=m \hbar \chi . k_{z}$ is the longitudinal wave vector of the full wave function. Upon integration, we see that

$$
U_{\text {lens }}=\exp \left(-i \frac{e^{2}}{8 \hbar^{2} k_{z}} \int_{-\infty}^{\infty} d z\left[B_{1}^{2}(z)-\frac{m \hbar}{e b^{2}} B_{3}(z)\right] \rho^{2}\right) .
$$

Comparing this with (1), we see that an initially collimated eigenstate of $L_{z}$ with quantum number $m$ that passes through the vector potential (3) will be focused at a distance $f_{m}$ from the center of the potential, where

$$
\frac{1}{f_{m}}=\frac{e^{2}}{8 m_{e} E} \int_{-\infty}^{\infty} B_{1}^{2}(z)-\frac{m \hbar}{e b^{2}} B_{3}(z) d z,
$$

where $E$ is the kinetic energy of the electron.

We can more simply rewrite this as

$$
f_{m}=\frac{f_{0}}{1-\Lambda m}
$$

where $f_{0}$ is the focal length of the $m=0$ eigenstate and the OAM dispersion coefficient $\Lambda=\frac{\beta_{0} \hbar}{e B_{0} b^{2}}$ is a dimensionless constant that depends only on the peak field strength $B_{0}$, the dispersion length $b$, fundamental constants, and a dimensionless $O(1)$ number $\beta_{0}$ that depends on the shape of the current distribution (Fig. 3). We calculate this focal length for several current distributions in Sec. III of the Supplemental Material [38]. For small OAM dispersion $\Lambda$, therefore, focal length is approximately linear with OAM.

$$
f_{m} \approx f_{0}(1+\Lambda m)
$$

(c) Physical realization of device. When the current source for the vector potential in (3) is a superconducting ring, there is an easy physical interpretation of the OAM dispersion coefficient $\Lambda$. A superconducting ring of radius $b$ encloses an area $\pi b^{2}$ and has an OAM dispersion coefficient inversely proportional to the number $n$ of flux quanta in the ring, as $n \propto \frac{B_{0} \pi b^{2}}{\Phi_{0}}$ and the flux quantum $\Phi_{0}=\frac{h}{2 e}$. We can therefore write the focal length of a lens made of a superconducting ring as

$$
f_{m} \approx f_{0}\left(1+\beta_{1} \frac{m}{n}\right),
$$

where $\beta_{1}$ is another $O(1)$ number.

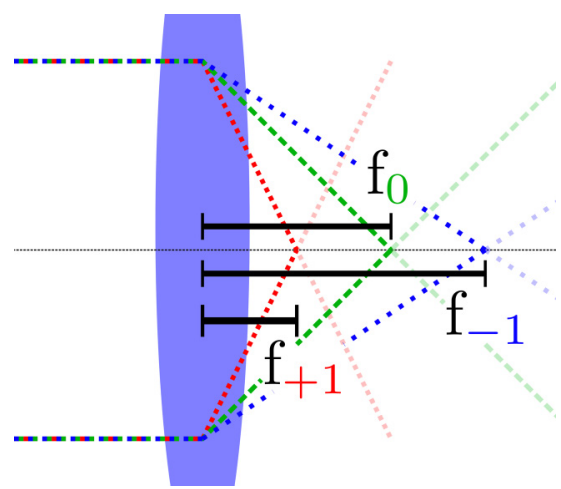

FIG. 3. Ray diagrams for a lens (blue disk) with a strongly OAMdependent focal length $f_{m}$ as given in (9). Red, dense dots: rays for $m=+1$ electrons; green dashes: rays for $m=0$ electrons; blue, loose dots: rays for $m=-1$ electrons. 

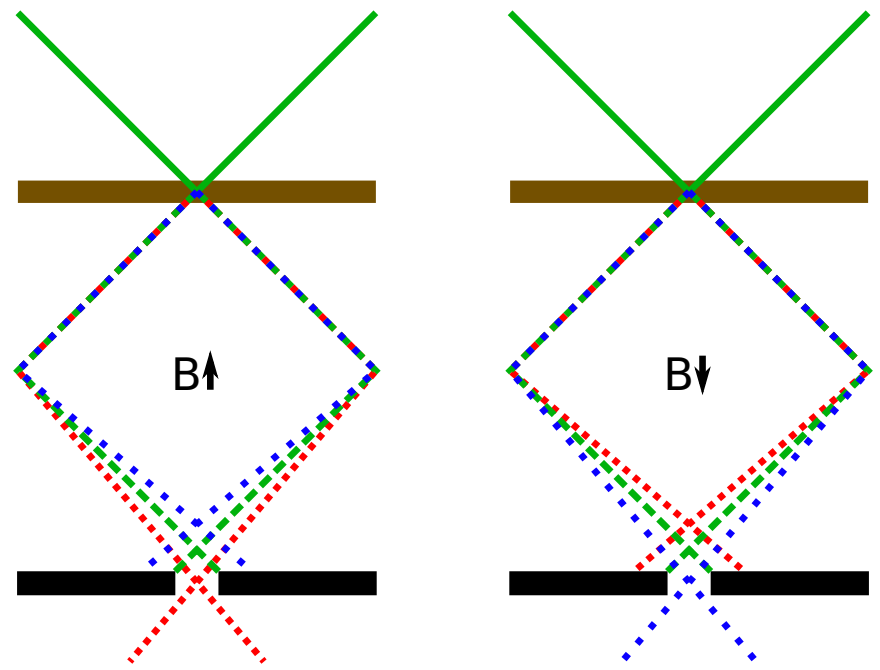

FIG. 4. Schematic ray diagrams for a dichroism experiment based on the OAM-dependent lensing effect. Interaction with a specimen (brown) produces a mix of outgoing $m=+1$ (red, dense dots), $m=0$ (green dashes), and $m=-1$ (blue, loose dots) OAM eigenstates. Left: An aperture (black) preferentially admits the $m=+1$ OAM eigenstate. The $m=+1$ state has a longer focal length in the positivepolarity lens (blue disk). Right: The aperture preferentially admits the $m=-1$ OAM state when the lens polarity is flipped.

If a lens can be constructed with a large OAM dispersion coefficient $\Lambda \sim 1$, the simplest application of this lensing effect to OAM measurement needs only a small aperture to select out one focused mode in the appropriate plane, as shown in Fig. 4. In fact, preferential admission of individual modes into an aperture might be used to perform a simple helical dichroism experiment without incident OAM. One can see that the focal length (7) has an OAM-independent part that depends just on the magnitude of the lensing magnetic field and an OAM-dependent part that depends on both the magnitude and direction of the magnetic field. In other words, one can control OAM dispersion via the direction of the lensing field. With an aperture set to preferentially admit the $m=+1$ mode, one can flip the polarity of the lens and therefore flip the sign of OAM dispersion, and consequentially admit the $m=-1$ mode without physically moving anything. This experiment likely will require an exceptionally stable microscope and careful alignment to ensure that no other beam properties change upon a lens polarity flip.

Several physical sources could produce a magnetic field with a significant OAM dispersion. The most obvious, but perhaps the most difficult to build, is a nanoscale solenoid. A solenoid with a radius on the order of $100 \mathrm{~nm}$ and a peak magnetic field on the order of 1 Tesla produces an OAM dispersion coefficient on the order of 0.1. The bound current density on the surface of a hole in an out-of-plane-polarized ferromagnetic thin film looks identical to the current density of a solenoid and could produce the same dispersion; such a hole would be far more easily nanofabricated and has the advantage over a loop of wire that the normal lensing effect will be partially canceled in the hole. A pulsed laser with a radially polarized magnetic field has the appropriate symmetry. The laser used in a recent experiment to prepare well-defined electron momentum states [43], with a peak magnetic field of $0.334 \mathrm{~T}$ and a spot size of $50 \mu \mathrm{m}$, would produce an OAM dispersion coefficient on the order of $10^{-6}$. This might be improved by several orders of magnitude with plasmonic field enhancement.

It is also likely that a more detailed analysis of nontrivial current distributions and electron propagation through them, including thick lens effects, could reveal other ways to maximize $B_{3}(z) / b^{2}$. In particular, for slower electrons, where adiabatic invariance of the magnetic moment can be assumed [44], it may be possible to analytically derive OAM-dependent effects in the propagation of electrons through a magnetic bottle or magnetic mirror.

A completely orthogonal approach to the realization of an OAM measurement device of this kind might involve stacking many lenses with a small OAM dispersion coefficient in a manner that magnifies the OAM-dependent effect. We discuss two possible designs for a stacked lens OAM measurement device below: an afocal system with spacing $2 f_{0}$ between lenses with opposite OAM dispersion, which produces exponentially increasing OAM-dependent magnification with number of lenses, as shown in Fig. 5; and a system with variable spacing between the lenses. Both designs produce strongly OAMdependent magnification but only weakly OAM-dependent image plane locations.

(d) Device design 1: Afocal system, or fixed separation between lenses. If the OAM dispersion coefficient $\Lambda=\frac{2 \hbar}{e B_{0} b^{2}}$ is small, then the focal length is approximately

$$
f_{m}=f_{0}(1+\Lambda m) \text {. }
$$

If we set two lenses back-to-back with a distance $2 f_{0}$ in between them with opposite OAM dispersion in each $\left(\Lambda_{1}=\right.$ $\left.-|\Lambda| ; \Lambda_{2}=|\Lambda|\right)$ we produce an afocal system with

$$
M_{m}=-(1+2 \Lambda m) \text {. }
$$

Since an afocal system produces no convergence or divergence-the effective focal length is infinite [45]—any combination of afocal systems is also an afocal system; this afocal system is thus easy to stack. In particular, for a stack of $N$ such afocal systems, in the limit of large $N$, the total

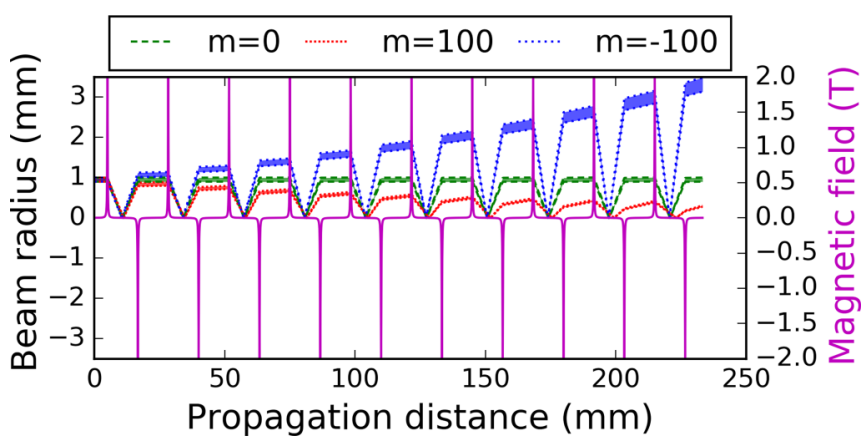

FIG. 5. Ray trajectories of $m=-100$ (blue), $m=0$ (green), and $m=+100$ (red) OAM modes propagating in a set of ten stacked afocal systems (13) of Glaser-model lenses (Eq. (50) in the Supplemental Material [38]) with longitudinal extent $a=100 \mu \mathrm{m}$, OAM dispersion length $b=1 \mu \mathrm{m}$, and maximum field strength $B_{0}=2 \mathrm{~T}$. The magnification of OAM goes exponentially with the number of lens sets. 


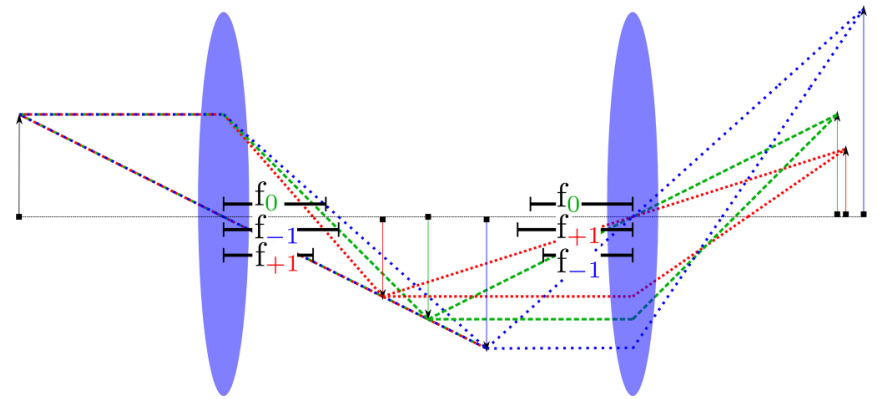

FIG. 6. Ray diagram for a combination of two lenses (blue disks) with variable spacing in between and opposite OAM dispersion which combine to produce a strongly OAM-dependent magnification, as given by (14) and a weakly OAM-dependent image position.

magnification approaches

$$
M_{m}^{N}=(-1)^{N} \exp (2 \Lambda m N) .
$$

This set of $N$ afocal systems has one clear advantage: even with arbitrarily small OAM dispersion $\Lambda$, we can easily distinguish between any two OAM orders with a sufficiently long stack $N$.

A 20-element $(N=20)$ set of identical afocal systems with $a=10 \mu \mathrm{m}, b=100 \mathrm{~nm}, B_{0}=2 \mathrm{~T}$, and a resultant $f_{0} \approx$ $60 \mathrm{~mm}$ has an OAM dispersion coefficient $|\Lambda|=0.066$ and a produces a magnification of an $m=+1,80 \mathrm{keV}$ electron beam of $\left|M_{+1}^{20}\right|=3.73$; on the other hand, an $m=-1$ beam sees a magnification of $\left|M_{-1}^{20}\right|=0.27$. A superposition of two otherwise-identical $m=+1$ and $m=-1$ modes passed through this device leave with a $14 \times$ difference in magnification. The total length of this device is on the order of a couple of meters.

(e) Device design 2: Variable spacing between lenses. If we place two lenses with opposite OAM dispersion back-to-back (Fig. 6) with a distance $2 \frac{s+1}{s} f_{0}$ between them, and place an object at a distance $(s+1) f_{0}$ in front of the first lens, we will see a focused image at a distance $(s+1) f_{0}$ behind the second lens with a magnification

$$
M_{m}=\frac{1}{1-2(s+1) \Lambda m} .
$$

The result is similar to that for an afocal system with two major differences: the advantage of this system is that larger magnification is produced by a larger spacing, rather than more lenses; the disadvantage is that only one mode can be fully separated from the rest at a time, as if $2(s+1) \Lambda m \approx 1$ so as to maximize magnification of the $m$-OAM components of the beam, then $2(s+1) \Lambda(m+1)$ cannot also be close to 1 unless $m$ is very large.

(f) Conclusion. If the measurement device can produce a sufficiently strong OAM-dependent magnification and the initial transverse spread of a state is small, the OAM distribution is mapped onto magnification. The orbital-angular-momentum spectrum can be quantitatively measured as the radial profile of the probability density after the electron is passed through the device. This OAM measurement is fully quantitative, parallel, and effective for inelastically scattered states.

As the orbital-angular-momentum-dependent lensing effect we study is a nondestructive measurement of OAM, it may also be possible to employ it for preparation of a pure OAM state. Pratical preparation of OAM states inevitably involves small errors in the definition of the correct amplitude and phase for a desired state. These small errors introduce extra OAM states in superposition with the desired state [11]. The combination of a single lens with a strong OAM-dependent lensing effect with a small aperture placed at the focal point of the desired OAM state is ideally suited to prepare pure OAM states.

We have demonstrated a Stern-Gerlach-like effect for measurement of electron orbital angular momentum. The measurement technique is applicable to the mixed states produced by inelastic scattering, which are otherwise difficult to measure. We outlined several strategies for practical implementation of this measurement device. If the device can be successfully built and integrated into electron spectrometers, simultaneous measurement of electron energy and orbitalangular-momentum distributions may be possible.

Acknowledgments. This work was partially supported by the U.S. Department of Energy, Office of Science, Basic Energy Sciences, under Award DE-SC0010466, and based in part on work supported by the National Science Foundation under Grant No. 1607733. Discussions with Christopher Jackson led to the quantum formalization of this idea. We appreciate careful scrutiny of the manuscript from Fehmi Yasin, Jordan Pierce, and Jordan Chess. We thank Martin Linck of Corrected Electron Optical Systems $\mathrm{GmbH}$ for order-of-magnitude estimates of lens focal lengths that were helpful in assessing practical relevance. Lastly, T.R.H. thanks Jordan Chess for many critical discussions.
[1] M. Uchida and A. Tonomura, Nature (London) 464, 737 (2010).

[2] J. Verbeeck, H. Tian, and P. Schattschneider, Nature (London) 467, 301 (2010).

[3] B. J. McMorran, A. Agrawal, I. M. Anderson, A. A. Herzing, H. J. Lezec, J. J. McClelland, and J. Unguris, Science 331, 192 (2011).

[4] K. Saitoh, Y. Hasegawa, N. Tanaka, and M. Uchida, J. Electron Microsc. 61, 171 (2012).

[5] P. Schattschneider, M. Stöger-Pollach, and J. Verbeeck, Phys. Rev. Lett. 109, 084801 (2012).
[6] A. M. Blackburn and J. C. Loudon, Ultramicroscopy 136, 127 (2014).

[7] A. Béché, R. Van Boxem, G. Van Tendeloo, and J. Verbeeck, Nat. Phys. 10, 26 (2014).

[8] T. R. Harvey, J. S. Pierce, A. K. Agrawal, P. Ercius, M. Linck, and B. J. McMorran, New J. Phys. 16, 093039 (2014).

[9] V. Grillo, G. C. Gazzadi, E. Karimi, E. Mafakheri, R. W. Boyd, and S. Frabboni, Appl. Phys. Lett. 104, 043109 (2014).

[10] R. Shiloh, Y. Lereah, Y. Lilach, and A. Arie, Ultramicroscopy 144, 26 (2014). 
[11] A. Béché, R. Juchtmans, and J. Verbeeck, Ultramicroscopy, (2016), doi:10.1016/j.ultramic.2016.05.006.

[12] P. Schattschneider, B. Schaffer, I. Ennen, and J. Verbeeck, Phys. Rev. B 85, 134422 (2012).

[13] A. Asenjo-Garcia and F. J. García de Abajo, Phys. Rev. Lett. 113, 066102 (2014).

[14] T. R. Harvey, J. S. Pierce, J. J. Chess, and B. J. McMorran, arXiv:1507.01810.

[15] Simulations [46] suggest that a small dichroism effect does exist when one measures only the probability density of the final state, and therefore traces out OAM in the final state.

[16] J. H. Macek, J. B. Sternberg, S. Y. Ovchinnikov, and J. S. Briggs, Phys. Rev. Lett. 104, 033201 (2010).

[17] S. J. Ward and J. H. Macek, Phys. Rev. A 90, 062709 (2014).

[18] J. M. Ngoko Djiokap, S. X. Hu, L. B. Madsen, N. L. Manakov, A. V. Meremianin, and A. F. Starace, Phys. Rev. Lett. 115, 113004 (2015).

[19] R. Géneaux, A. Camper, T. Auguste, O. Gobert, J. Caillat, R. Taïb, and T. Ruchon, Nat. Commun. 7, 12583 (2016).

[20] V. Serbo, I. P. Ivanov, S. Fritzsche, D. Seipt, and A. Surzhykov, Phys. Rev. A 92, 012705 (2015).

[21] R. Takahashi and N. Nagaosa, Phys. Rev. B 91, 245133 (2015).

[22] M. Schüler, J. Berakdar, and Y. Pavlyukh, Phys. Rev. A 92, 021403 (2015).

[23] E. Karimi, L. Marrucci, V. Grillo, and E. Santamato, Phys. Rev. Lett. 108, 044801 (2012).

[24] I. P. Ivanov, Phys. Rev. D 85, 076001 (2012).

[25] J. Yuan, S. M. Lloyd, and M. Babiker, Phys. Rev. A 88, 031801 (2013).

[26] G. Guzzinati, L. Clark, A. Béché, and J. Verbeeck, Phys. Rev. A 89, 025803 (2014).

[27] L. Clark, A. Béché, G. Guzzinati, and J. Verbeeck, Phys. Rev. A 89, 053818 (2014).

[28] R. Shiloh, Y. Tsur, R. Remez, Y. Lereah, B. A. Malomed, V. Shvedov, C. Hnatovsky, W. Krolikowski, and A. Arie, Phys. Rev. Lett. 114, 096102 (2015).
[29] M. Shutova, A. A. Zhdanova, and A. V. Sokolov, Phys. Lett. A 381, 408 (2017).

[30] T. Yahn, J. Pierce, T. Harvey, and B. McMorran, Microsc. Microanal. 19, 1166 (2013).

[31] K. Saitoh, Y. Hasegawa, K. Hirakawa, N. Tanaka, and M. Uchida, Phys. Rev. Lett. 111, 074801 (2013).

[32] W. Gerlach and O. Stern, Z. Phys. 9, 349 (1922).

[33] E. Noether, Transp. Theory Stat. Phys. 1, 186 (1971).

[34] H. Batelaan, T. J. Gay, and J. J. Schwendiman, Phys. Rev. Lett. 79, 4517 (1997).

[35] H. Rose, Geometrical Charged-Particle Optics (Springer, New York, 2013).

[36] L. Reimer and H. Kohl, Transmission Electron Microscopy: Physics of Image Formation (Springer, New York, 2008).

[37] J. V. Neumann, Mathematical Foundations of Quantum Mechanics (Princeton University Press, Princeton, NJ, 1955).

[38] See Supplemental Material at http://link.aps.org/supplemental/ 10.1103/PhysRevA.95.021801 for comprehensive derivations (Secs. I and II), calculation of the OAM-dependent focal length for several specific lens models (Sec. III), and discussion of spherical aberration (Sec. IV) and its correction (Sec. V) in relation to OAM-dependent lensing.

[39] V. Grillo, L. Marrucci, E. Karimi, R. Zanella, and E. Santamato, New J. Phys. 15, 093026 (2013).

[40] The nonunitarity of the lowest-order approximation to the transformation induced by $L_{z}$ terms produces a small, unphysical loss of probability density with each slice.

[41] P. Schattschneider, V. Grillo, and D. Aubry, Ultramicroscopy 174, 8 (2017).

[42] G. Pozzi, Ultramicroscopy 30, 417 (1989).

[43] A. Feist, K. E. Echternkamp, J. Schauss, S. V. Yalunin, S. Schäfer, and C. Ropers, Nature (London) 521, 200 (2015).

[44] H. Alfven, Cosmical Electrodynamics (Clarendon Press, Oxford, 1963).

[45] D. Malacara-Hernández and Z. Malacara-Hernández, Handbook of Optical Design, 2nd ed. (CRC Press, Boca Raton, 2014).

[46] J. Rusz, J.-C. Idrobo, and S. Bhowmick, Phys. Rev. Lett. 113, 145501 (2014). 